\title{
A Low-Loss Substrate-Independent Approach for 60-GHz Transceiver Front-End Integration Using Micromachining Technologies
}

\author{
Bo Pan, Member, IEEE, Yuan Li, Student Member, IEEE, George E. Ponchak, Fellow, IEEE, \\ Manos M. Tentzeris, Senior Member, IEEE, and John Papapolymerou, Senior Member, IEEE
}

\begin{abstract}
This paper presents a low-loss, substrate-independent approach to integrate transceiver front-ends for $60-\mathrm{GHz}$ wireless applications. Dielectric loss is eliminated by using polymer and bulk silicon micromachining technologies to create a cavity-based duplexer and a horn antenna in the air, above the substrate. A coplanar waveguide input is used for easy integration of the low-noise amplifier and power amplifier of the receiver and transmitter, respectively, with the micromachined passive module. A prototype is designed, fabricated, and characterized, with the transmit band (TX) set between $58.7-59.5 \mathrm{GHz}$ and the receive band (RX) as 60.6-61.4 GHz. The proposed method offers an easy integration of both planar components and 3-D integrated modules on top of the substrate.
\end{abstract}

Index Terms-Cavity resonator filter, millimeter wave, on-wafer pattern measurement, silicon bulk micromachining, 60-GHz, SU-8, surface micromachining.

\section{INTRODUCTION}

$\mathbf{R}$ ESEARCH and development activities for $V$-band (60-GHz) wireless personal area networks (WPANs) for broadband and high-data-speed multimedia applications are expanding because of the $7 \mathrm{GHz}$ of unlicensed spectrum that exists in many countries. Significant progress has been made in the development of individual circuits and passive components [1]-[3]. However, very few papers have reported integration and packaging of a whole transceiver front-end [4]-[7]. Packaging and integration of passive components with active circuits is challenging at $V$-band because of incompatible substrate requirements, parasitic reactance caused by bond wires and via holes, and increased losses (dielectric and conductor).

There are two approaches to address this issue. In [1], [4], and [8], an on-chip antenna is integrated with the integrated circuits onto a silicon substrate to reduce the system size and increase

Manuscript received May 13, 2008; revised September 12, 2008. First published November 18, 2008; current version published December 05, 2008. This work was supported in part by the Georgia Research Alliance and the U.S. Army Research Office.

B. Pan was with the School of Electrical and Computer Engineering, Georgia institute of Technology, Atlanta, GA 30332 USA. He is now with Realtek Semiconductor, Irvine, CA 92602 USA (e-mail: bo.pan@ realtek.com).

Y. Li, M. M. Tentzeris, and J. Papapolymerou are with the School of Electrical and Computer Enineering, Georgia Insitute of Technology, Atlanta, GA 30332 USA (e-mail: yuanli@ece.gatech.edu; etentze@ece.gatech.edu; papapol@ece. gatech.edu).

G. E. Ponchak is with theNASA Glenn Research Center, Cleveland, $\mathrm{OH}$ 44135 USA (e-mail: george.ponchak@ieee.org).

Color versions of one or more of the figures in this paper are available online at http://ieeexplore.ieee.org.

Digital Object Identifier 10.1109/TMTT.2008.2007358 the integration simplicity. However, the antenna gain was reported to be from -7 to $-9 \mathrm{dBi}$, which is very low and severely degrades the system performance. This low gain may require another stage in the amplifier, which decreases the system efficiency. Furthermore, dipole antennas have a narrow bandwidth.

Integration of a high-performance antenna into a package or on a separate substrate provides another realistic solution. In [5] and [7], antennas are integrated on low-loss substrates that are prohibitively expensive for commercial applications, and the whole design is optimized from a system-on-package (SoP) point of view. Antenna gains as high as 10 and $7 \mathrm{dBi}$ are measured, respectively. Substrate-integrated-waveguide (SIW) antennas with various forms, including an SIW horn antenna, a tapered slot antenna backed by an SIW cavity, and SIW slot arrays, have been studied in [9]-[11], respectively. They also require low-loss and low permittivity substrates for antenna integration. These solutions might not be of interest to the system-on-chip (SoC) community since a specially chosen substrate is required for antenna integration. In previous research, we demonstrated significant performance improvement by elevating several individual millimeter-wave components into the air above the substrate, including antennas, couplers, and filters [12], [13], using SU-8 based surface micromachining technologies [15], [16].

In this paper, we report for the first time a full-duplex $60-\mathrm{GHz}$ transceiver front-end module, including the passive components (the antenna and duplexer) and the amplifiers (a transmit power amplifier and a receive low-noise amplifier) shown in Fig. 1. The duplexer and antenna are fabricated on a carrier substrate using SU-8 micromachining fabrication processes. The amplifiers are surface-mounted onto the carrier substrate, but they may be monolithically fabricated on the substrate. Surface mounting of the ICs creates a substrate-independent solution. A proof-of-concept prototype is designed, fabricated and characterized, with the transmitting band (TX) set between $58.7-59.5 \mathrm{GHz}$ and the receiving band (RX) as 60.6-61.4 GHz. First, the cavity filter duplexer design and characterization is shown followed by the horn antenna design and characterization. Finally, the measured characteristics of the passive transceiver and the active transceiver are shown and compared.

\section{SyStem DESIGN}

For a $58.7-59.5-\mathrm{GHz}$ TX band and a 59.6-60.4-GHz RX band transceiver with high data rates, a high-gain $(>10 \mathrm{dBi})$ 


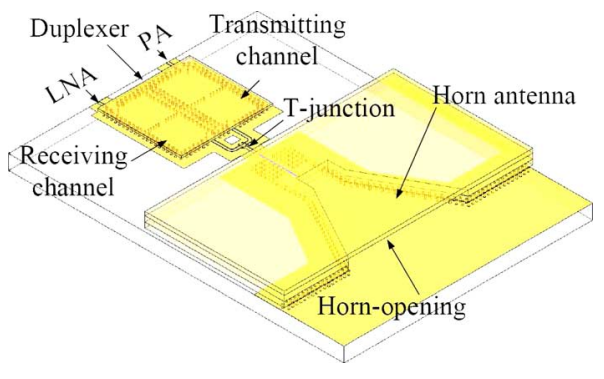

Fig. 1. Schematic of the transceiver front-end integration.

integrated antenna is required due to the increased signal attenuation attributed to $\mathrm{O}_{2}$ absorption. In addition, the antenna needs to be broadband to cover the entire range from 57 to $64 \mathrm{GHz}$ while maintaining its radiation pattern characteristics throughout this range. A micromachined $V$-band horn antenna discussed in [17] is selected as the ideal candidate for this purpose.

The duplexer consists of two narrowband bandpass filters in parallel. A high quality factor is required for the resonant cavities to assure low insertion loss in the passband and high rejection in the stopband while maximizing the isolation between the two channels. For this reason, the surface-micromachined pillars that will form the cavities must have an appropriately designed height. The dependence of the quality factor of such cavities to the height of pillars are extensively studied in [13] and not repeated in this paper.

To optimize the transceiver module, a final design is performed that considers various integration issues. The transceiver front-end module shown in Fig. 1 is integrated onto a 1-in $\times$ 1.5-in glass wafer. Fig. 2 shows the layout of the micromachined passive components and the integrated amplifiers on the glass substrate. Fig. 2(a) shows the cross-sectional view of the whole integration, with the major dimensions marked in Fig. 2(b). Passive modules are formed by CPW lines on the surface of a substrate, rows of pillars that have cured SU-8 cores, and stacked silicon pieces bonded on top of pillars. The unpackaged amplifiers are surface-mounted to the top surface of the substrate using silver epoxy and then connected to the passive modules using bond wires. The assembly method is for the sole purpose of prototyping here; in a massive production stage, active circuitry could be monolithically fabricated on the silicon/ compound substrates or flip chip die attached so that no wirebonding is necessary.

\section{Component Design}

\section{A. V-Band Duplexer}

A duplexer, which must separate an input signal into two or more output channels and provide high isolation between channels, is a key component for a transceiver front-end. It can be realized by putting two bandpass filters in parallel. In practice, the center frequencies of these two filters are very close. Thus, to get high isolation between channels, filters with very sharp roll-off skirts are required. For a planar-type filter, this can be realized by either increasing the order of the filter, leading to

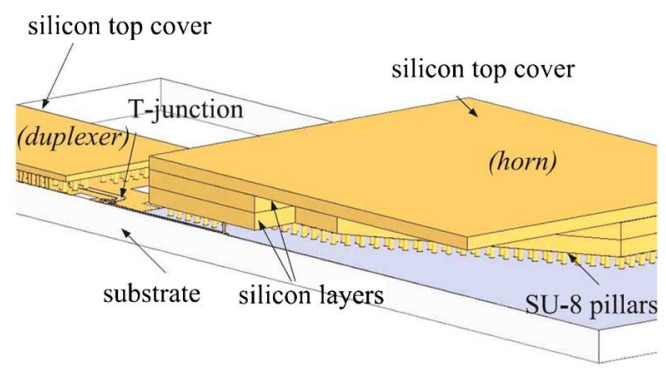

(a)

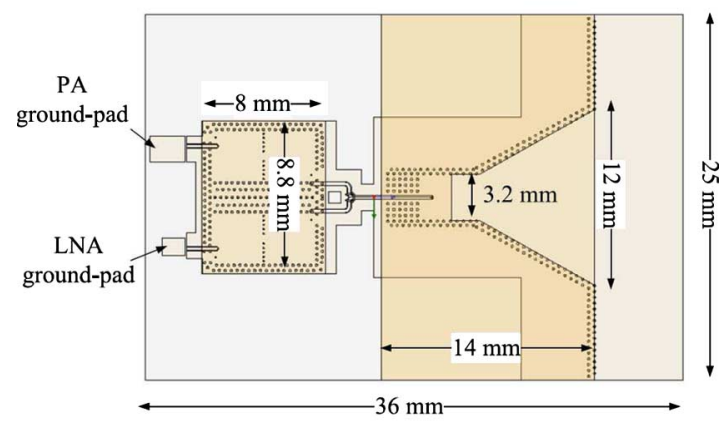

(b)

Fig. 2. (a) Cross-sectional view of the transceiver integration. (b) Top view of the transceiver module with major dimensions marked.

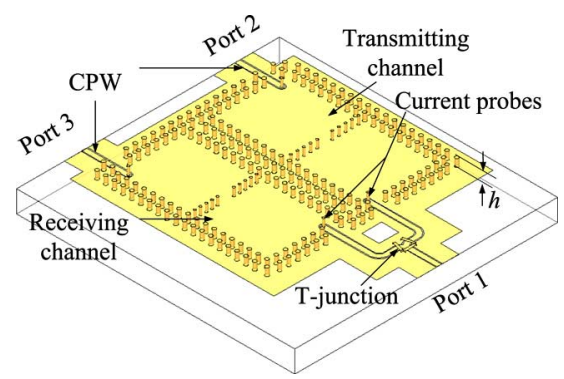

Fig. 3. Schematic of the micromachined duplexer (top cover not shown for clarity).

a large number of cascaded resonators, or by introducing multiple transmission zeros in the stopband. Still, the out-of-band rejection is quite limited since the unloaded quality factor of a planar resonator is not sufficiently high. Another important requirement for a duplexer is low insertion loss for both channels, which improves the overall receiver sensitivity and reduces the overall power consumption of the transceiver. For a planar filter, this requirement can be met by using thick metals, superconducting metals, enclosed packages to reduce radiation, and substrates with a lo loss tangent. A duplexer based on cavity filters reported in [13] is an ideal solution since it has very low radiation loss and it is not dependent on the substrate. Here, design techniques and experimental results for a surface micromachined duplexer are reported.

1) Duplexer Design: As shown in Fig. 3, the duplexer using the surface-micromachining technology consists of two twopole filters and a T-junction that connects the two filters. Port 2 is the output of the power amplifier and Port 3 is the input to the LNA, while Port 1 is reserved for the common antenna. The two-pole filter uses a direct probe-feed described in [13]. 
TABLE I

OPTIMIZED DimENSIONS OF TwO-POLE TRANSMISSION ZERO FILTERS (UNITS: MILLIMETERS)

\begin{tabular}{|c|c|c|}
\hline Design Parameters & Transmitting channel & Receiving channel \\
\hline cavity length $\left(l_{c}\right)$ & 3.64 & 3.64 \\
cavity width $\left(w_{c}\right)$ & 3.64 & 3.42 \\
iris position $\left(y_{i}\right)$ & 1.48 & 1.35 \\
iris length $(i)$ & 0.69 & 0.74 \\
probe position $\left(X 1_{o f f}\right)$ & 0.54 & 0.52 \\
probe position $\left(Y 1_{o f f}\right)$ & 0.35 & 0.39 \\
probe position $\left(X 2_{o f f}\right)$ & 0.59 & 0.50 \\
probe position $\left(Y 2_{o f f}\right)$ & 0.39 & 0.39 \\
iris pillar pitch $\left(p_{i}\right)$ & 0.30 & 0.30 \\
iris pillar diameter $\left(d_{i}\right)$ & 0.10 & 0.10 \\
side pillar pitch $\left(p_{s}\right)$ & 0.40 & 0.40 \\
side pillar diameter $\left(d_{s}\right)$ & 0.17 & 0.17 \\
\hline
\end{tabular}

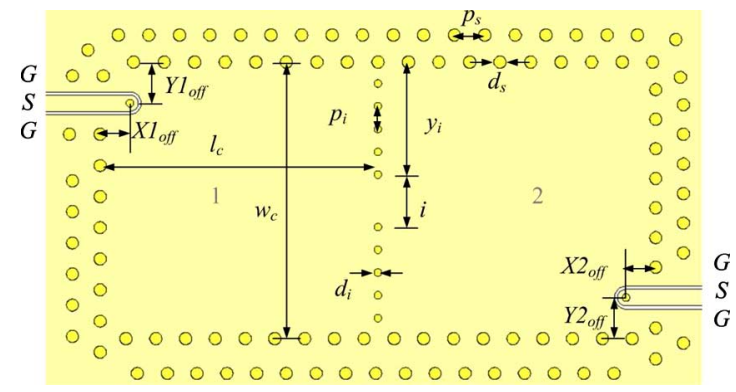

Fig. 4. Physical meanings of duplexer design parameters.

The filter design procedure is described in [13] and is similar as the one used for SIW-based filters [14], [18]. The center frequencies of the two channels are 59.1 and $61.0 \mathrm{GHz}$. The $3-\mathrm{dB}$ bandwidth is chosen as $0.8 \mathrm{GHz}$ for both channels. Larger than $20-\mathrm{dB}$ return loss is required to minimize power reflection at the I/O ports. Compared with the design in [13], a narrow bandwidth is chosen for good channel selectivity. Table I lists the optimized parameters for the two filters, and the physical meanings of all parameters are provided in Fig. 4. Full-wave simulation predicts a $1.92-\mathrm{dB}$ insertion loss and larger than $20-\mathrm{dB}$ return loss for both filters.

To achieve high channel-to-channel isolation, the design of the CPW line T-junction shown in Fig. 5 is critical. While it is possible to design a waveguide-based T-junction to directly connect a waveguide horn antenna, thus avoiding a waveguide-CPW-waveguide transition which increases fabrication complexity and introduces additional transition loss. The only reason to use the waveguide-CPW-waveguide configuration is to enable the characterization of all of the individual components using a probe station, while using the same set of masks.

For the T-junction shown in Fig. 5, an appropriate length of $L_{1}$ is required to present an open circuit condition looking into channel 1 at the T-junction at the resonating frequency of channel 2. Similarly, the length of $L_{2}$ is adjusted to transform the output impedance of channel 2 into an open circuit at the resonating frequency of channel 1. Mathematically, this leads to the following design equation:

$$
L_{1} \approx \frac{n \lambda_{g 2}}{2} \quad L_{2} \approx \frac{n \lambda_{g 1}}{2}
$$

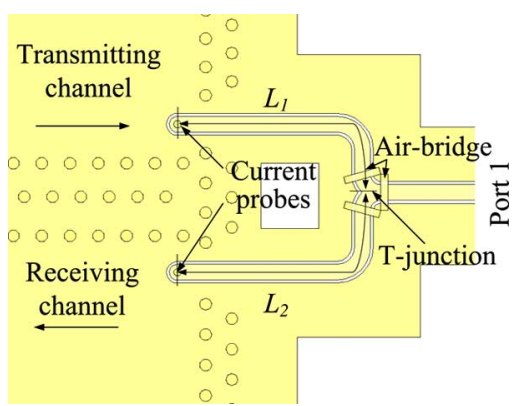

Fig. 5. Schematic of the CPW T-junction.

TABLE II

OPTIMIZED SIMULATION RESULTS FOR A DUPLEXER

\begin{tabular}{|c|c|c|}
\hline Optimized results & Transmitting channel & Receiving channel \\
\hline matching & $>15 \mathrm{~dB}$ & $>15 \mathrm{~dB}$ \\
insertion loss & $3.05 \mathrm{~dB}$ & $3.14 \mathrm{~dB}$ \\
isolation & $>35 \mathrm{~dB}$ & $>35 \mathrm{~dB}$ \\
\hline
\end{tabular}

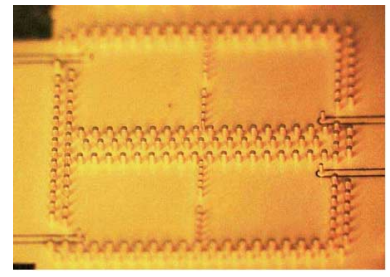

(a)

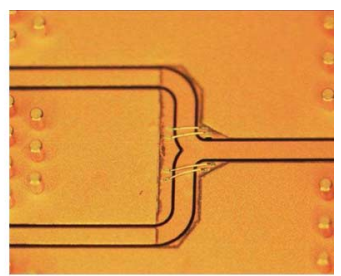

(b)
Fig. 6. Micro-images of the fabricated duplexer

where $\lambda_{g 1}$ and $\lambda_{g 2}$ are the effective resonant wavelengths of channels 1 and 2, respectively. This equation only gives the initial design value for the length of each arm in the T-junction; optimization is required to compensate for the parasitic reactance of the T-junction and 90 degree bend and to account for layout requirements. The line lengths are first chosen by using Agilent-ADS schematic simulations with a data box that contains the full-wave simulation result of the duplexer. After line lengths are determined with this method, a full-wave simulation by Ansoft HFSS is performed to extract the scattering matrix of the T-junction. The final optimized values of $L_{1}$ and $L_{2}$ are 3.34 and $3.50 \mathrm{~mm}$, respectively.

Simulation results of an optimized duplexer design are plotted together with measurement results and will be discussed in detail in next subsection. Table II summarizes the simulated duplexer performance.

2) Duplexer Fabrication and Measurement: Fig. 6 shows pictures of the fabricated sample before a top cover is bonded. To prevent slot mode radiation from the T-junction, the CPW ground planes are connected together by bonding wires. The scattering parameters of this duplexer are measured on an 8510 $\mathrm{XF}$ network analyzer. The unused port is terminated by a broadband coaxial load on a probe. To facilitate the probe orientation during measurement, three sets of duplexers with different CPW pad orientation are fabricated.

Figs. 7-9 show the simulated and measured scattering parameters of the duplexer. In general, good agreement between simulation and measurement is observed. The insertion loss for 


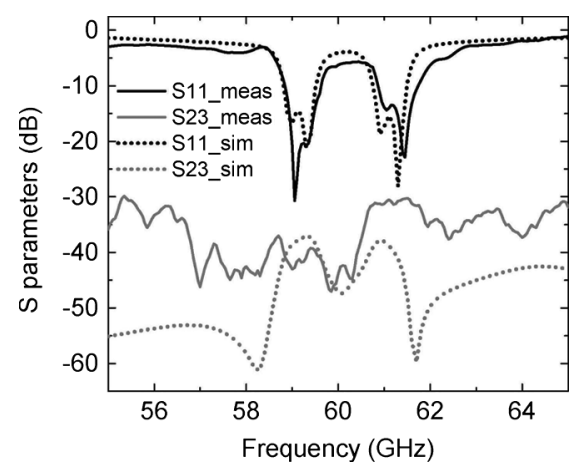

Fig. 7. Simulated and measured duplexer performance: impedance matching at the power combining port, and isolation between two channels.

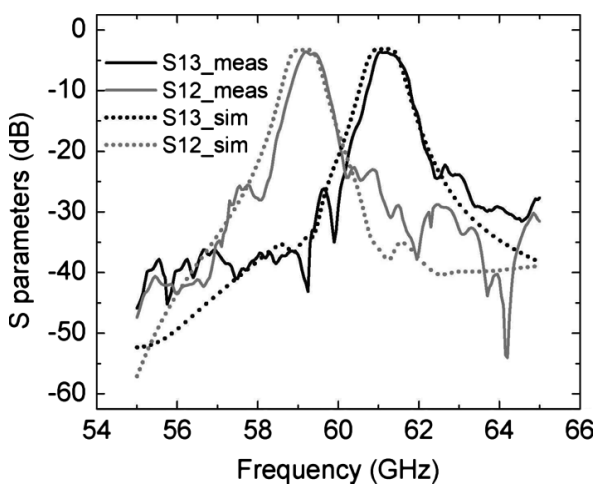

Fig. 8. Simulated and measured duplexer performance: insertion loss for each channel.

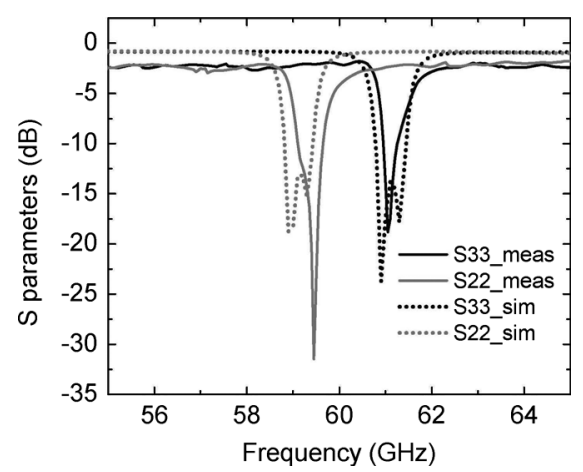

Fig. 9. Simulated and measured duplexer performance: impedance matching at power dividing ports.

channels 1 and 2 is 3.58 and $3.60 \mathrm{~dB}$, respectively, which is approximately $1 \mathrm{~dB}$ larger than the simulated insertion loss. The center frequencies of the two channels are both shifted upwards approximately $0.5 \%$, the maximal measured return loss is larger than $15 \mathrm{~dB}$ for all three ports, with a return loss of larger than $10 \mathrm{~dB}$ observed for the band of interest, and the isolation is larger than $30 \mathrm{~dB}$ across the whole band. Slight discrepancies between measurement and simulation, such as the center frequency shift and impedance mismatch, can be observed. They are mainly attributed to several non-ideal fabrication conditions, including variation of pillars' height, variation of the diameters
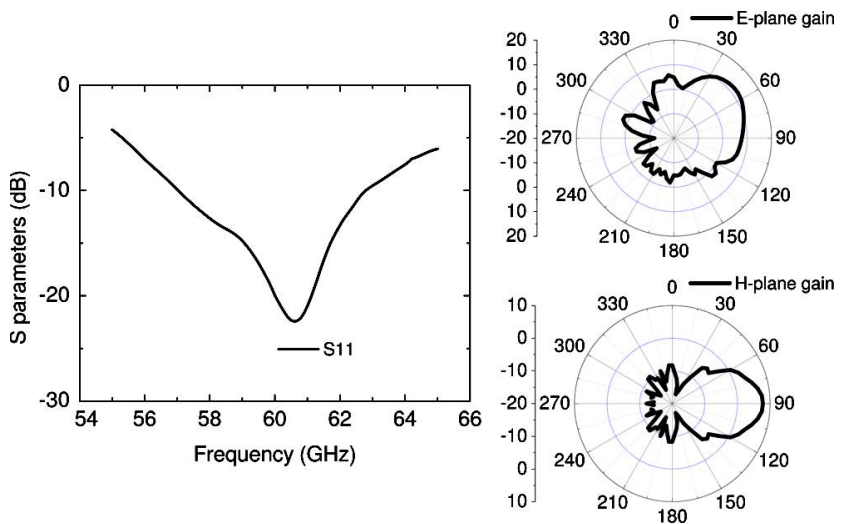

Fig. 10. Simulated horn performance.

of pillars, and failure of a few pillars. The details will be discussed in Section V.

\section{B. Antenna Development for Integration}

The micromachined horn structure is similar to the one described in [17] and optimized for the transceiver. It is composed of three parts: a CPW-connected vertical current probe for feeding, a rectangular waveguide with a height of $0.35 \mathrm{~mm}$ to help transition the CPW mode to the waveguide mode, and an $H$-plane linearly flared horn as shown in Fig. 2. A linear flare in the $E$-plane is hard to realize in this technology, so a simple $E$-plane step from 0.35 to $1.4 \mathrm{~mm}$ at the horn opening is used instead. The CPW-connected vertical current probe directly contacts the rectangular waveguide on top of the substrate. Four rows of pillars are used to terminate the back-short to ensure there is no direct wave leakage into the backside. Two rows of pillars with staggered positions are used as the waveguide sidewalls, as well as the lower part of the horn sidewall. Two $525 \mu \mathrm{m}$-thick metalized silicon pieces are stacked on top of the pillar arrays to build the horn's upper part. One solid metalized silicon piece is used as the horn's top wall.

The optimized horn is $14 \mathrm{~mm}$ long and $12 \mathrm{~mm}$ wide, with a backshort of $0.9 \mathrm{~mm}$ long and $3.2 \mathrm{~mm}$ wide. The $H$-plane flare angle is $58^{\circ}$. The $E$-plane step is located $1.3 \mathrm{~mm}$ after the vertical probe, and the overall height of this horn is $1.4 \mathrm{~mm}$.

The simulated return loss for the optimized horn antenna is plotted in Fig. 10, where it is seen that it is larger than $10 \mathrm{~dB}$ from $57 \mathrm{GHz}$ to $63 \mathrm{GHz}$. Also shown in Fig. 10 are the simulated $E$ - and $H$-plane radiation patterns. As can be seen, since the horn flare is only on the $\mathrm{H}$-plane, the $30^{\circ} \mathrm{H}$-plane beam width is much narrower than the $50^{\circ} \mathrm{E}$-plane beamwidth. The radiation pattern is tilted into the upper space on the $E$-plane as a result of wave reflection from the 6-mm metal strip extended from the bottom wall of the horn that is seen in Fig. 2. The maximum gain is $11.5 \mathrm{dBi}$, and the front-to-back ratio is $13 \mathrm{~dB}$.

A picture of a fabricated horn lower part is shown in Fig. 11. The horn's measured characteristics are not shown here since this topology has been verified in [17] and it is directly integrated with the duplexer. 


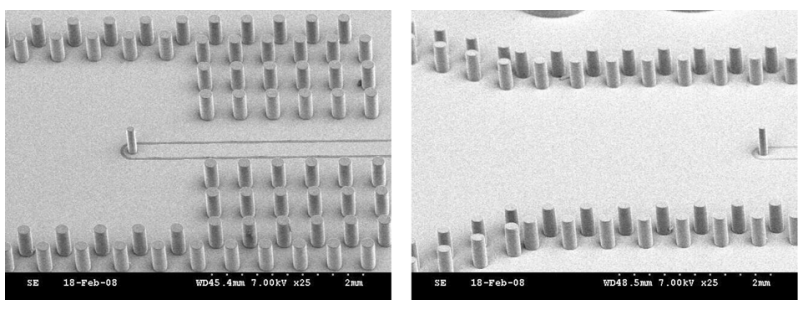

Fig. 11. Micro-images of a fabricated horn.

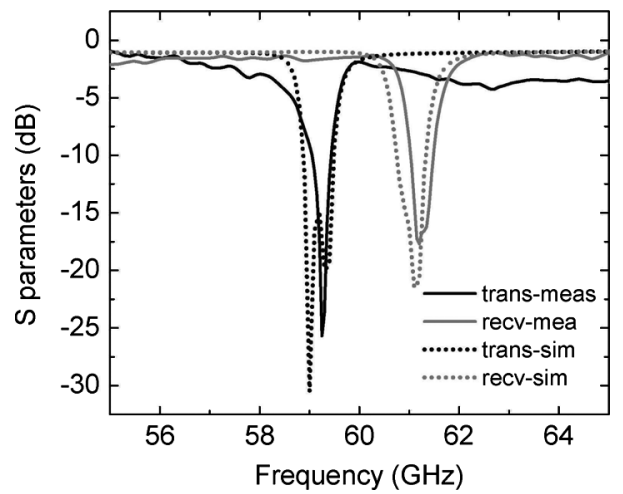

Fig. 12. Simulated and measured input matching of a transceiver front-end passive module.

\section{TRANSCEIVER FRONT-END INTEGRATION}

\section{A. Duplexer/Antenna Integration}

Integration of the duplexer and the antenna is straightforward because their I/O interfaces are both CPW lines. To prevent damaging sidewall pillars during wire-bonding at the CPW T-junction, a 1.5-mm-long section of CPW is used between the two components.

The simulated and measured scattering parameters of the two-port integrated module are shown in Fig. 12 where good agreement is seen. The measured isolation is better than $26 \mathrm{~dB}$ across the frequency range of interest, and the measured return loss is greater than $10 \mathrm{~dB}$ for both channels. These results demonstrate that the duplexer/antenna section of the transceiver have the desired characteristics.

\section{B. Amplifier Integration}

As a substrate-independent solution, the polymer-core conductor surface micromachining technology can provide a versatile platform for system integration. Ideally, to demonstrate this advantage, all passive components discussed above should be integrated directly above an active circuit die. In this scenario, all modules are connected with regular transmission lines or vias between different metal layers. This integration scheme introduces minimal parasitics associated with a bonding wire or solder bumps. However, developing active circuits at this frequency, though available both in academia and industry, is far from mature. Therefore, commercially available, unpackaged amplifier chips from the Hittite Company are used.

A low-noise amplifier (LNA), HMC-ALH382, is die- attached in the receiving channel and a medium power amplifier (PA), HMC-ABH209, is die attached in the transmitting channel. Before the RF input/output is wire-bonded to CPW

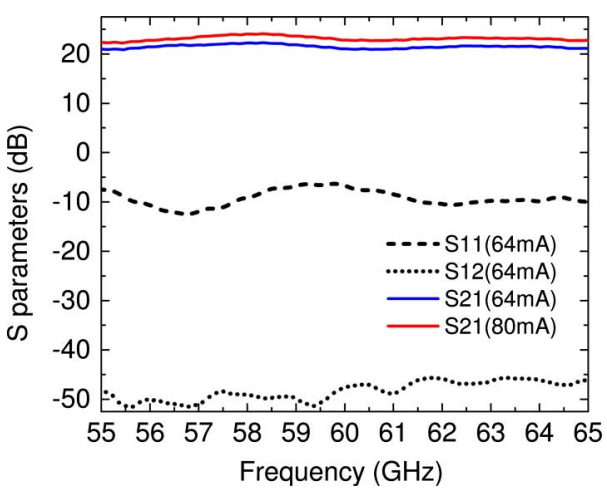

Fig. 13. Measured LNA performance for different biasing current.

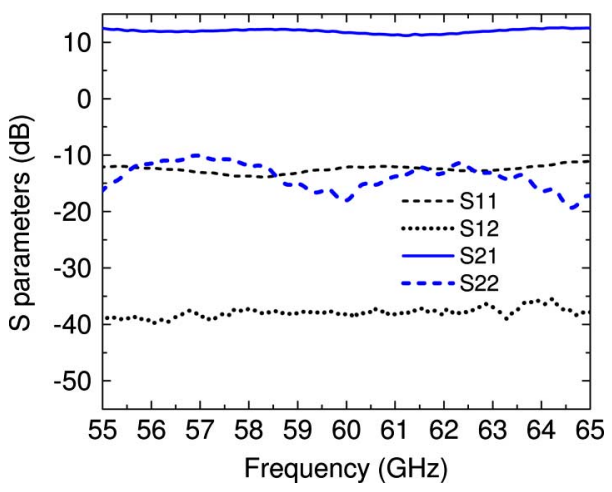

Fig. 14. Measured PA performance.

lines that feed the duplexer, the amplifiers were tested on chip using dc and RF probes. Fig. 13 shows the measured LNA scattering parameters. As can be seen, a total $64 \mathrm{~mA}$ drain current gives a forward gain of $21 \mathrm{~dB}$, an average return loss of $8 \mathrm{~dB}$, and a reverse isolation of greater than $45 \mathrm{~dB}$; increasing the total drain current to $80 \mathrm{~mA}$ increases the gain by $2 \mathrm{~dB}$. The measured on-chip scattering parameters for the PA are shown in Fig. 14, where an average gain of $12 \mathrm{~dB}$ is observed.

The amplifiers are then wire-bonded to the transceiver. $\mathrm{Al} / \mathrm{Si}$ bonding wires with $1.5 \mathrm{mil}(38 \mu \mathrm{m})$ diameter are used to connect the RF pads of the amplifiers with the CPW I/O lines. To minimize reflections, the $220 \mu \mathrm{m} \mathrm{CPW}$ signal line width is tapered to $100 \mu \mathrm{m}$ while maintaining the same characteristic impedance. The length of bonding wire and its associated inductance is critical for impedance matching. Although shorter bond wires are generally preferred in the RF path, based on our best bonding facility and expertise, a length of approximately $350 \mu \mathrm{m}$ is used for all RF bonding.

DC biasing circuits are patterned onto a 4 mil $(100 \mu \mathrm{m})$ thick, LCP substrate. Two decoupling capacitors of $100 \mathrm{pF}$ and $0.1 \mu \mathrm{F}$ are attached to the LCP and used for the gate and drain biasing. The biasing circuitry on LCP is bonded on top of the glass substrate using silver epoxy. Interconnects between dc pads on the amplifier chip and pads on the LCP circuit are realized again using wire-bonding. Here, long wire bonds are used to provide a large inductance for RF choking. Fig. 15 shows a micro-image of a LCP dc bias circuit and amplifier chip.

Fig. 16 shows the integrated transceiver front-end before the lid is attached. The lid is manually bonded to the pillar-array 


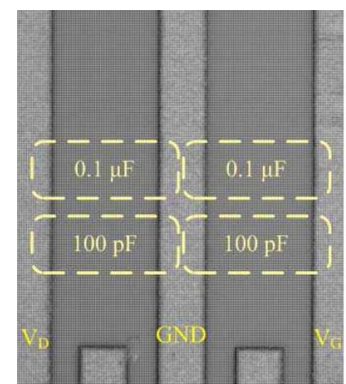

(a)

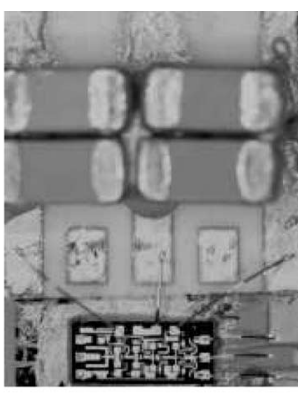

(b)
Fig. 15. Biasing circuits on a 4-mil LCP.

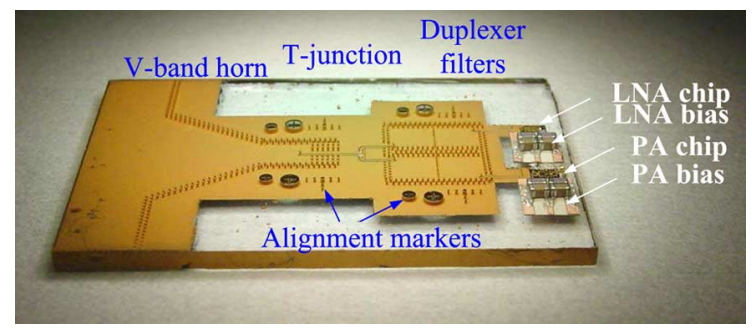

Fig. 16. Integration of amplifiers, duplexer, and antenna before bulk-micromachined upper half is bonded.

sidewalls using high-conductivity nanoparticle silver epoxy [20]. No alignment is needed for this step. To prevent performance degradation, silver epoxy is only applied to top ends of pillars by carefully flipping the circuit and dipping all pillar ends into a layer of shallow silver epoxy. It is very important to avoid directly applying silver epoxy on the metalized silicon cover.

Next, bulk-micromachined silicon pieces for the horn upper half are bonded with the aid of a brass fixture. The brass fixture is used to align and hold together the bulk-micromachined top and bottom (Fig. 16) layers since alignment between layers is critical for performance; manually bonding with silver epoxy is not a good choice here. In this prototype assembly, the brass fixture we used has a precisely machined cover and four screws. By adjusting the pressure using the four screws, the air gap between different layers can be removed. In a massive production, gaps between different layers can be avoided when a thermal-compression substrate bonding tool is available. Fig. 17(a) shows the integrated module after duplexer cover is bonded and dry-etched silicon pieces are assembled; Fig. 17(b) shows the final integrated module after two brass covers are fastened to press all layers tightly.

\section{Wireless Transmission Testing}

Wireless transmission tests are performed before amplifiers are integrated and after, respectively. For a test without amplifier, an integrated module with duplexer and antenna is connected to Port 1 of an 8510 XF network analyzer, while a standalone $\mathrm{CPW}$-fed micromachined horn antenna reported in [17] is connected to Port 2. S21/S12 is plotted to test wireless transmission between the two modules. TRL calibration is performed and the unused port is terminated with $50 \mathrm{Ohm}$ load using a probe positioner. Both the transmission channel and the receiving channel are tested and results are plotted in Figs. 18

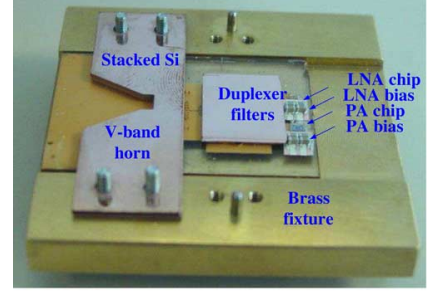

(a)

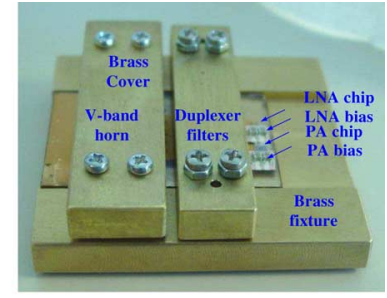

(b)
Fig. 17. (a) Integration picture after duplexer cover is bonded and bulk-micromachined horn upper half is assembled. (b) Final assembled circuit with the aid of a brass fixture.

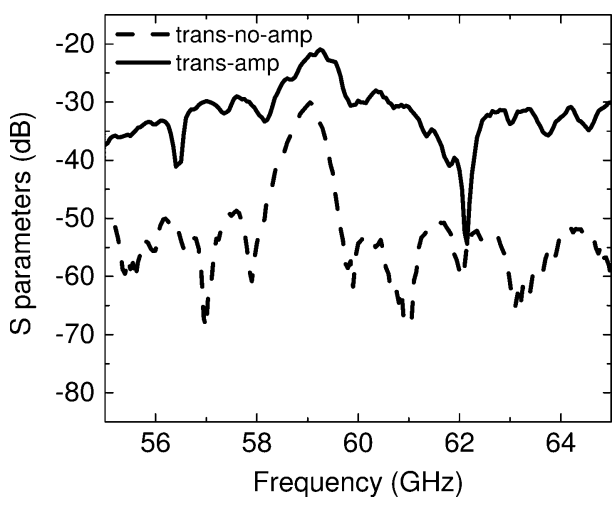

Fig. 18. Measured wireless test results for transmission channel.

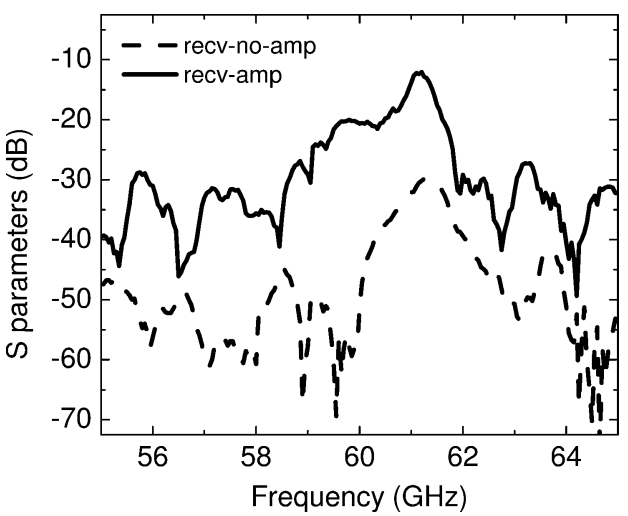

Fig. 19. Measured wireless test results for receiving channel.

and 19, respectively. As can be seen, wireless transmission peaks are found at 59.2 and $61.25 \mathrm{GHz}$, respectively.

After the amplifiers are integrated, wireless transmission test is performed again. Connection between the modules and the network analyzer is the same except that the unused port is not terminated with a $50-\Omega$ load. This is because the amplifier needs three dc probes for biasing and there is no space for an additional RF probe From the amplifier test, we found that the amplifier input/output is matched to $50 \Omega$ even with dc pads all connected in floating mode. The transmit and receive characteristics are plotted in Figs. 18 and 19 respectively, with comparison of results without amplifier.

Transmission peaks are observed at almost the same frequencies as the passive system. Comparing the transmitted power between the active and passive circuits in each channel, it is seen that the active channel is $10 \mathrm{~dB}$ higher in the TX mode and $18 \mathrm{~dB}$ 


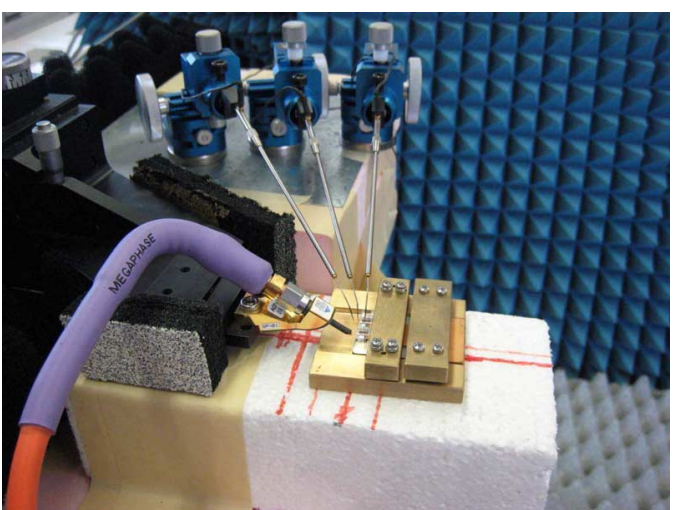

Fig. 20. Specialized radiation pattern and gain measurement setup; only probes and foam stage shown here.

higher in the RX mode. Comparing these results with the amplifier $S$-parameters measured on chip and shown in Figs. 13 and 14, it is seen that only 2 and $3 \mathrm{~dB}$ of loss in the two channels, respectively, results from the die attach, wire bonds, dc bias circuit, and mismatch to the duplexer.

\section{Radiation Pattern and Gain Measurement}

After completion of $S$-parameter measurements, the channel-2 LNA was damaged and replaced by a PA for radiation pattern and gain measurements. The transceiver is characterized on a specialized far field antenna range comprised of an RF GSG probe head supported on a wood/foam shelf, a Styrofoam shelf to support the antenna under test, a rotary stage that rotates a WR-15, $25 \mathrm{dBi}$ gain horn in an arc around the antenna under test, and an Agilent $67 \mathrm{GHz}$ PNA. DC probes for biasing the amplifier are supported by the same shelf that supports the RF probe head. The radius of the arc traversed by the gain horn, which is the distance between the gain horn and the antenna under test, is $57 \mathrm{~cm}$. The system is calibrated with a second, identical WR-15, $25 \mathrm{dBi}$ gain horn. The measured results are not corrected for the difference in insertion loss between the $1.85 \mathrm{~mm}$ to WR-15 adapter required for the calibration and the probe at $60 \mathrm{GHz}$. Cascade estimates the insertion loss of the probe at $60 \mathrm{GHz}$ to be $2 \mathrm{~dB}$, and the insertion loss of the adapter is estimated to be $1.2 \mathrm{~dB}$. Also, the M/A Com data sheets estimate the gain of the horn antenna to be $0.2 \mathrm{dBi}$ lower at $60 \mathrm{GHz}$. Thus, overall, there could be $+0.6 \mathrm{~dB}$ correction to the measured gain, but because these insertion loss and gain corrections were not verified, the data was not corrected. Instead, it is stated that the error in the measured data is estimated to be $0.6 \mathrm{~dB}$. Ripples on the measured radiation patterns are due to required auxiliary equipment, such as microscope stand and microscope, metal probe fixture, metal stand to support the wood/foam shelf, and metal sheet required to hold the dc probe heads; absorber was used to minimize the effects of the metal structures but it could not eliminate them. Fig. 20 shows RF probe, dc probes and foam stage to support DUT for pattern/gain measurement.

Fig. 21 shows measured $E$-plane co-polarization and crosspolarization gain. The maximum total gain is read as $14.5 \mathrm{dBi}$ at approximately $\theta=55^{\circ}$.

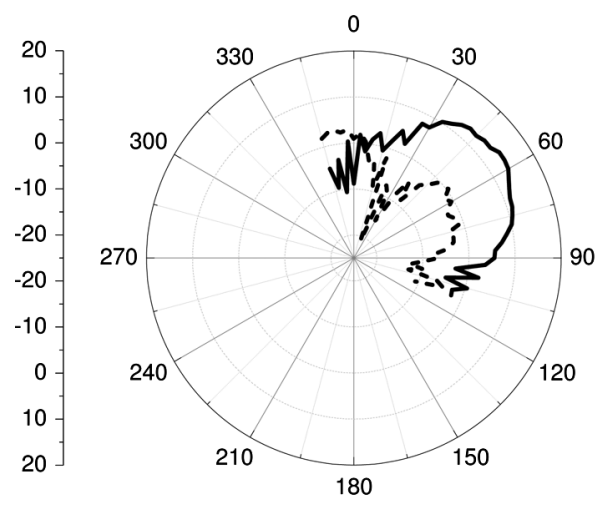

-Measured E-plane co-pol with amplifier - - Measured E-plane cross-pol with amplifier

Fig. 21. Measured $E$-plane copolarization and crosspolarization gain patterns.

TABLE III

THEORETICAL GAIN CALCULATION BREAKDOWN

\begin{tabular}{|c|c|c|c|c|c|}
\hline & amplifier & duplexer & line loss & horn antenna & overall \\
\hline gain & $10 \mathrm{~dB}$ & $-3.6 \mathrm{~dB}$ & $-1.1 \mathrm{~dB}$ & $11.5 \mathrm{~dB}$ & $16.8 \mathrm{~dB}$ \\
\hline
\end{tabular}

Table III calculates the expected gain from this measurement. The amplifier's gain is estimated as $10 \mathrm{~dB}$ based on wireless transmission testing data shown in Fig. 18. Various line losses are estimated as $1.1 \mathrm{~dB}$ based on our characterization data of CPW lines on this glass substrate. Since this integrated horn antenna is not characterized separately, its theoretical gain is used in calculating overall gain of the integrated module. The calculated gain is $16.8 \mathrm{~dB}$. Considering another additional $+0.6 \mathrm{~dB}$ correction stated previously and the measured antenna gain could be lower than its theoretical value, good agreement is achieved between calculation and measurement.

\section{DisCUSSIONS}

\section{A. Frequency Limitations}

In terms of the frequency limitation of this approach, we believe that this approach is able to cover the whole submillimeter-wave band up to $300 \mathrm{GHz}$. This prediction is based on the assumption that the active circuits have already been integrated onto a wafer and a packaging company or a semiconductor manufacturing company adopts this approach as a post process to form passive waveguide-based components.

The standard photolithographic process can form features down to several micron with a pretty good yield, so the challenge does not lie in the photolithography itself. The specific material SU-8, used here to build smaller pillars, is not the limit either; this is because the most important thing to make a reliable pillar is to maintain relatively low aspect ratio of the pillar. Researchers have demonstrated that 10-25 aspect-ratios can be realized. Here, we only use $2-4$. We have observed very good mechanical reliability. For a $300-\mathrm{GHz}$ application, the length or width of an air cavity is about $700 \mu \mathrm{m}$. The diameters and pitch of pillars shrink to $40 \mu \mathrm{m}$ and $70-80 \mu \mathrm{m}$, respectively, to accommodate this dimension. With these sizes, pillar's height up to about $150 \mu \mathrm{m}$ can be easily patterned. The corresponded 


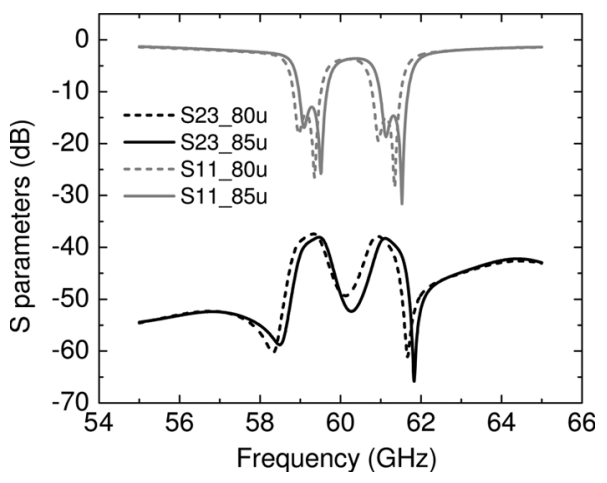

Fig. 22. Scattering parameters versus the diameters of sidewall pillars.

quality factor (around 480 in our EM simulation) is still far better than the one by directly printing a planar resonator on top of a doped wafer.

With the proximity alignment used in this technique, alignment error sets an upper boundary for the frequency. When the operating frequency goes higher, the whole structure size shrinks, thus the maximal allowed misalignment becomes less. For pillars of $40 \mu \mathrm{m}$, separated with $70-80 \mu \mathrm{m}, 5-10 \mu \mathrm{m}$ is the maximal misalignment allowance.

\section{B. Fabrication Complexity vs. Performance Improvement}

In terms of benefits compared with the introduced complexity, performance and manufacturing accuracy still overweigh other factors. At $60 \mathrm{GHz}$ and beyond, traditional machined waveguide parts are costly due to a stringent fabrication accuracy requirement. They are also bulky due to the existence of flanges and are difficult to integrate. The higher the frequency, the more expensive of the traditional machined waveguide parts. In contrast, this proposed solution should become cheaper as the frequency goes up with reduced material consumption and reduced numbers of silicon wafer stacking.

\section{Discrepancies Between Simulations and Measurements}

We have observed slight discrepancies between simulation and measurement results in both the duplexer and the transceiver front-end. Post-fabrication EM simulations have attributed these discrepancies mainly to the following three factors. Only S11 and S23 of the duplexer are shown here as an example due to the page limitation. Similar effects are observed for other parameters.

1) Variation of the Radius of Side-Wall Pillars: As shown in Fig. 22, the frequency response shifts when the diameters of the sidewall pillars are changed (in most cases they are enlarged while keeping the pillars' original positions). This happens during the formation of the pillars by introducing an increased exposure to make the pillars mechanically strong.

2) Variation of the Height of Pillars: Height control of the SU-8 coating is another critical point of the fabrication. As shown in Fig. 23, varying the SU-8 pillars' height will not result in a frequency shift, but it could change the quality factor of the cavity, thus introducing a slight mismatch at the $\mathrm{I} / \mathrm{O}$ ports.

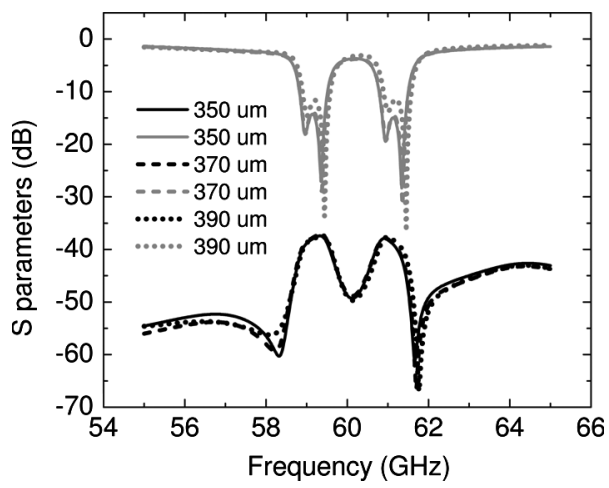

Fig. 23. Scattering parameters versus the height of pillars.

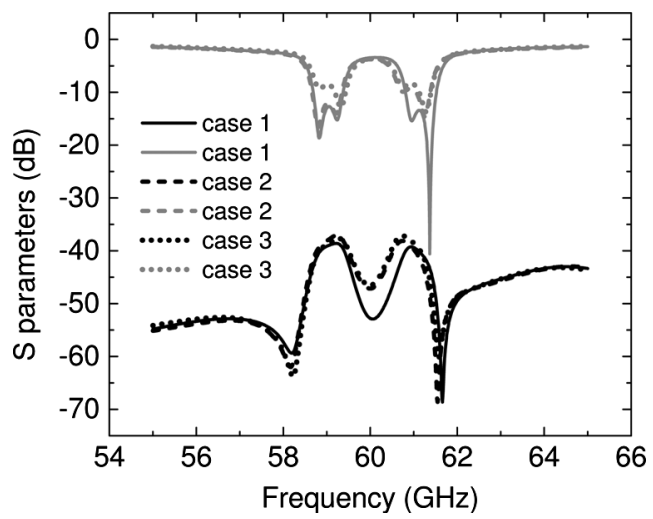

Fig. 24. Scattering parameters variation while removing a few pillars.

3) Failure of Pillars: We have observed a great yield of SU-8 pillars during our fabrication. However, a few pillars might fail because of various human errors during assembly and testing. Fig. 24 shows EM simulations results when we randomly remove 1-2 SU-8 pillars from the inner wall of the cavity. As can be seen from Fig. 24, pillar failure can result in impedance mismatch.

\section{CONCLUSION}

In this paper, a novel approach to integrate high performance millimeter-wave transceiver front-end using polymer-core conductor surface micromachining technology is presented for the first time. By elevating a cavity-filter-based duplexer and a horn antenna on top of the substrate and using air as the filler, the dielectric loss can be eliminated. A full-duplex transceiver front-end integrated with amplifiers are designed, fabricated, and comprehensively characterized to demonstrate advantages brought by this surface-micromachining technology. The proposed method offers an easy integration of both planar components and 3-D integrated modules on top of the substrate. It is a low loss and substrate-independent solution for millimeter-wave transceiver integration.

\section{ACKNOWLEDGMENT}

The authors would like to thank the reviewers and the editors for their very professional comments and suggestions, which greatly helped improve the manuscript quality. The authors would also like to thank Dr. D. Zhao and L. Rose for providing 
assistance in wirebonding, Dr. Y. Yoon, Dr. Y. Choi, and Prof. M. Allen for advice on optimizing the polymer-core-conductor fabrication flow.

\section{REFERENCES}

[1] B. Razavi, "CMOS transceivers at $60 \mathrm{GHz}$ and beyond," in Proc. Int. Symp. Circuits Syst., May 27-30, 2007, pp. 1983-1986.

[2] J.-H. Lee, N. Kidera, S. Pinel, J. Laskar, and M. Tentzeris, "Fully integrated passive front-end solutions for a V-band LTCC wireless system," IEEE Antennas Wireless Propag. Lett., vol. 6, pp. 285-288, 2007.

[3] R. Bairavasubramanian, S. Pinel, J. Laskar, and J. Papapolymerou, "Compact $60-\mathrm{GHz}$ bandpass filters and duplexers on liquid crystal polymer technology," IEEE Microw. Wireless Compon. Lett., vol. 16, no. 5, pp. 237-239, May 2006.

[4] T. Mitomo, R. Fujimoto, N. Ono, R. Tachibana, H. Hoshino, Y. Yoshihara, Y. Tsutsumi, and I. Seto, "A 60-GHz CMOS receiver front-end with frequency synthesizer," IEEE J. Solid-State Circuits, vol. 43, no. 4, pp. 1030-1037, Apr. 2008.

[5] U. Pfeiffer, J. Grzyb, D. Liu, B. Gaucher, T. Beukema, B. Floyd, and S. Reynolds, "A chip-scale packaging technology for $60-\mathrm{GHz}$ wireless chipsets," IEEE Trans. Microw. Theory Tech., vol. 54, no. 8, pp. 3387-3397, Aug. 2006.

[6] T. Zwick, D. Liu, and B. Gaucher, "Broadband planar superstrate antenna for integrated millimeterwave transceivers," IEEE Trans. Antennas Propag., vol. 54, no. 10, pp. 2790-2796, Oct. 2006.

[7] S. Sarkar and J. Laskar, "A single-chip 25 pj/bit multi-gigabit $60 \mathrm{GHz}$ receiver module," in IEEE MTT-S Int. Microw. Symp. Dig. , Jun. 3-8, 2007, pp. 475-478.

[8] M. Sanduleanu and J. Long, "CMOS integrated transceivers for $60 \mathrm{GHz}$ UWB communication," in Proc. IEEE Int. Conf. Ultra-Wideband, Sep. 24-26, 2007, pp. 508-513.

[9] W.-G. Yeo, T.-Y. Seo, J.-W. Lee, and C.-S. Cho, "H-plane sectoral filtering horn antenna in PCB substrates using via fences at millimetrewave," in Proc. 37th Eur. Microw. Conf., Munich, Germany, Oct. 2007, pp. 818-821.

[10] I.-K. Kim, N. Kidera, S. Pinel, J. Papapolymerou, J. Laskar, J.-G. Yook, and M. M. Tentzeris, "A linear tapered cavity-backed slot antenna for millimeter-wave LTCC modules," IEEE Antennas Wireless Propag. Lett., vol. 5, pp. 175-178, Dec. 2006.

[11] L. Yan, W. Hong, G. Hua, J. Chen, K. Wu, and T. J. Cui, "Simulation and experiment on SIW slot array antennas," IEEE Microw. Wireless Compon. Lett., vol. 14, no. 9, pp. 446-448, Sep. 2004.

[12] B. Pan, Y. Yoon, P. Kirby, J. Papapolymerou, M. Tenzeris, and M. Allen, "A $W$-band surface micromachined monopole for low-cost wireless communication systems," in IEEE MTT-S Int. Microw. Symp. Dig., Jun. 6-11, 2004, vol. 3, pp. 1935-1938.

[13] B. Pan, Y. Li, M. M. Tentzeris, and J. Papapolymerou, "High performance surface micromachined millimeter-wave cavity filters," IEEE Trans. Microw. Theory Tech., vol. 56, no. 4, pp. 959-970, Apr. 2008.

[14] J.-H. Lee, N. Kidera, G. DeJean, S. Pinel, J. Laskar, and M. Tentzeris, "A $V$-band front-end with 3-D integrated cavity filters/duplexers and antenna in LTCC technologies," IEEE Trans. Microw. Theory Tech., vol. 54, no. 7, pp. 2925-2936, Jul. 2006.

[15] M. Despont, H. Lorenz, N. Fahrni, J. Brugger, P. Renaud, and P. Vettiger, "High-aspect-ratio, ultrathick, negative-tone near-UV photoresist for MEMS applications," in Proc. IEEE 10th Annu. Int. Workshop Micro Electro Mechanical Syst., Jan. 26-30, 1997, pp. 518-522.

[16] Y.-K. Yoon, J.-W. Park, and M. G. Allen, "Polymer-core conductor approaches for RF MEMS," J. Microelectromechan. Syst., vol. 14, pp. 886-894, Oct. 2005.

[17] B. Pan, Y. Li, G. E. Ponchak, J. Papapolymerou, and M. M. Tentzeris, "A high-gain broadband surface micromachined $V$-band horn," IEEE Trans. Antennas Propag., submitted for publication.

[18] D. Deslandes and K. Wu, "Accurate modeling, wave mechanisms, and design considerations of substrate integrated waveguide," IEEE Trans. Microwave Theory Tech., vol. 54, no. 6, pp. 2516-2526, Jun. 2006.

[19] C. Balanis, Antenna Theory, 2nd ed. New York: Wiley, 1997, pp. 678-678.

[20] H. Jiang, K. Moon, Y. Li, and C. P. Wong, "Surface functionalized silver nanoparticles for ultra-highly conductive polymer composites," Chem. Mater., vol. 18, no. 13, pp. 2969-2973, 2006.

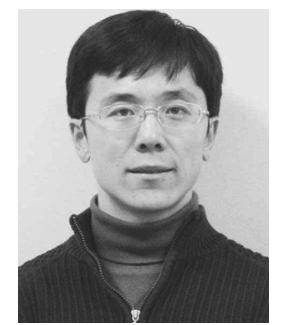

Bo Pan (S'04-M'08) received the B.S. and M.S. degrees from Tsinghua University, Beijing, China, in 2000 and 2003, respectively, and the Ph.D. degree from the Georgia Institute of Technology, Atlanta, in 2008.

He was a Graduate Research Assistant with the Georgia Institute of Technology MiRCTECH and ATHENA research groups, the Georgia Electronic Design Center (GEDC), and the GT-NSF Packaging Research Center (PRC). He is currently with Realtek Semiconductor Group, Wionics Research, Irvine, CA. He has published more than 30 papers in refereed journals and conference proceedings and two book chapters. His research at the Georgia Institute of Technology involved the design, fabrication, integration, and characterization of various passive components and circuits for millimeter-wave T/R front ends, with the focus on micromachining/MEMS technologies. His current research is IC packaging design and phased arrays for $60-\mathrm{GHz}$ wireless personal area networks (WPAN) applications. He is also working on the design of embedded UWB antennas for various UWB products.

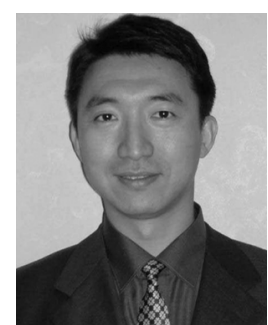

Yuan Li (S'05) received the B.S. degree in automatic control from the Northwestern Polytechnical University, China, in 1994, the M.S. degree in automatic control from the Nanjing University of Aeronautics and Astronautics, China, in 1997, and the M.S in electrical engineering from the Georgia Institute of Technology, Atlanta, in 2004. He is currently working toward $\mathrm{Ph} . \mathrm{D}$. degree in electrical and computer engineering at the Georgia Institute of Technology.

Before beginning his doctoral work, he was an Electronic and RF Engineer in industry. $\mathrm{He}$ is currently a Research Assistant with the Georgia Institute of Technology MiRCTECH Research Group, the Georgia Electronic Design Center (GEDC), Atlanta. His research interests include the development and characterization of micromachined circuits for millimeter-wave and terahertz applications, packaging technology for millimeter-wave systems, and passive and active circuits for wireless systems.

Mr. Li was the recipient of the 2007 IEEE Microwave Theory and Techniques Society Graduate Fellowship Award.

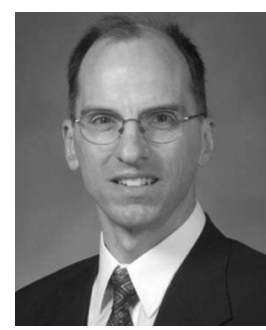

George E. Ponchak (S'82-M'83-SM'97-F'08) received the B.E.E. degree from Cleveland State University, Cleveland, OH, in 1983, the M.S.E.E. degree from Case Western Reserve University, Cleveland, in 1987, and the Ph.D. degree in electrical engineering from the University of Michigan, Ann Arbor, in 1997.

He joined the staff of the Communication Technology Division, NASA Glenn Research Center, Cleveland, in 1983, where he is now a Senior Research Engineer. In 1997-1998 and in 2000-2001, he was a Visiting Professor with Case Western Reserve University. He has authored and coauthored over 150 papers in refereed journals and symposia proceedings. His research interests include the development and characterization of microwave and millimeter-wave printed transmission lines and passive circuits, multilayer interconnects, uniplanar circuits, $\mathrm{Si}$ and $\mathrm{SiC}$ radio frequency integrated circuits, and microwave packaging.

Dr. Ponchak is an associate member of the European Microwave Association. $\mathrm{He}$ is editor-in-chief of the IEEE MICROWAVE AND WIRELESS COMPONENTS LETTERS, and he was editor of a special issue of the IEEE TRANSACTIONS ON Microwave THEORY AND TECHNIQUES on Si MMICs. He founded the IEEE Topical Meeting on Silicon Monolithic Integrated Circuits in RF Systems and served as its Chair in 1998, 2001, and 2006. He served as Chair of the Cleveland MTT-S/AP-S Chapter (2004-2006), and he has chaired many symposium workshops and special sessions. He is a member of the IEEE International Microwave Symposium Technical Program Committee on Transmission Line Elements and served as its Chair in 2003-2005 and a member of the IEEE Microwave Theory and Techniques Society (MTT-S) Technical Committee 12 on Microwave and Millimeter-Wave Packaging and Manufacturing. He served on the IEEE MTT-S Administrative Committee (AdCom) Membership Services Committee (2003-2005). He was the recipient of the Best Paper of the ISHM'97 30th International Symposium on Microelectronics Award. 


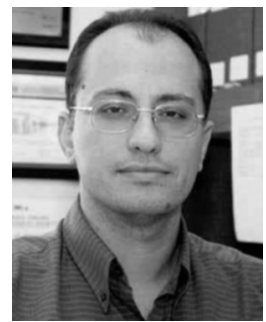

Manos M. Tentzeris (S'89-M'98-SM'03) received the B.S.E.E degree from the National Technical University of Athens, Athens, Greece, and the M.S. and Ph.D. degrees in electrical engineering and computer science from The University of Michigan at Ann Arbor.

$\mathrm{He}$ is currently an Associate Professor with the School of Electrical and Computer Engineerign, Georgia Institute of Atlanta (Georgia Tech), Atlanta. He has published more than 290 papers in refereed journals and conference proceedings, three books, and ten book chapters. He has helped develop academic programs in highly integrated/multilayer packaging for RF and wireless applications using ceramic and organic flexible materials, paper-based RFIDs and sensors, microwave MEMs, SOP-integrated antennas, and adaptive numerical electromagnetics and heads the ATHENA Research Group (20 researchers). He is the Associate Director and the leader of the RFID Research Group of the Georgia Electronic Design Center (GEDC) of the State of Georgia.

Dr. Tentzeris is a member of URSI-Commission D, a member of MTT-15 committee, an Associate Member of EuMA, a Fellow of the Electromagnetic Academy and a member of the Technical Chamber of Greece. He was the recipient/corecipient of the 2007 IEEE APS Symposium Best Student Paper Award, the 2007 IEEE IMS Third Best Student Paper Award, the 2007 ISAP 2007 Poster Presentation Award, the 2006 IEEE Microwave Theory and Techniques (MTT) Outstanding Young Engineer Award, the 2006 Asian-Pacific Microwave Conference Award, the 2004 IEEE TRANSACTIONS ON ADVANCED PACKAGING Commendable Paper Award, the 2003 NASA Godfrey "Art" Anzic Collaborative Distinguished Publication Award, the 2003 IBC International Educator of the Year Award, the 2003 IEEE CPMT Outstanding Young Engineer Award, the 2002 Georgia Tech-ECE Outstanding Junior Faculty Award, the 2001 ACES Conference Best Paper Award, and the 2000 National Science Foundation CAREER Award. He was also the 1999 Technical Program Co-Chair of the 54th ARFTG Conference and the Chair of the 2005 IEEE CEM-TD Workshop. He will be the TPC Chair for IEEE IMS 2008 Symposium. He is an Associate Editor of the IEEE TRANSACTIONS ON MiCROWAVE THEORY AND TECHNIQUES, the IEEE TRANSACTIONS ON ADVANCED PACKAGING, and the International Journal on Antennas and Propagation.

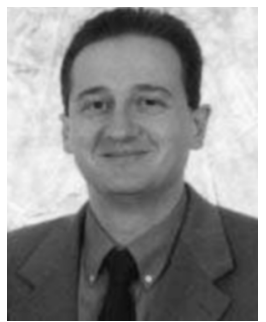

John Papapolymerou (S'90-M'99-SM'04) received the B.S.E.E. degree from the National Technical University of Athens, Athens, Greece, in 1993, and the M.S.E.E. and Ph.D. degrees from The University of Michigan at Ann Arbor in 1994 and 1999 , respectively.

From 1999 to 2001, he was an Assistant Professor with the Department of Electrical and Computer Engineering, University of Arizona, Tucson. In 2001, he joined the School of Electrical and Computer Engineering of the Georgia Institute of Technology, Atlanta, where he is currently an Associate Professor. He has authored or coauthored over 200 publications in peer-reviewed journals and conferences. His research interests include the implementation of micromachining techniques and MEMS devices in microwave, millimeter-wave, and THz circuits and the development of both passive and active planar circuits on semiconductor $(\mathrm{Si} /$ $\mathrm{SiGe}$ and GaAs) and organic substrates (LCP and LTCC) for system-on-a-chip/ system-on-a-package RF front-ends.

Dr. Papapolymerou is the vice-chair for Commission D of the U.S. National Committee of URSI and as an associate editor for the IEEE MICROWAVE AND WIRELESS COMPONENT LETTERS and the IEEE TRANSACTIONS ON ANTENNAS and Propagation. During 2004, he was the Chair of the IEEE Microwave Theory and Techniques (MTT)/Antennas and Propagation (AP) Atlanta Chapter. He was the recipient of the 2004 Army Research Office (ARO) Young Investigator Award, the 2002 National Science Foundation (NSF) CAREER Award, the Best Paper Award at the 3rd IEEE International Conference on Microwave and Millimeter-Wave Technology (ICMMT2002), Beijing, China, and the 1997 Outstanding Graduate Student Instructional Assistant Award presented by the American Society for Engineering Education (ASEE), The University of Michigan Chapter. His students have also been recipients of several awards, including the Best Student Paper Award of the 2004 IEEE Topical Meeting on Silicon Monolithic Integrated Circuits in RF Systems, the 2007 IEEE MTT-S Graduate Fellowship, and the 2007-08 IEEE MTT-S Undergraduate Scholarship/Fellowship. 